Title:

\title{
MTHFD1 1958 G>A genetic polymorphism (rs2236225) dichotomy in schizophrenia: lower manifestation risks, but more severe negative symptoms
}

Running title:

\section{Role of MTHFD1 1958 G>A genetic polymorphism (rs2236225) in schizophrenia}

Zhilyaeva T.V., Chekanina O.M., Rukavishnikov G.V., Blagonravova A.S., Mazo G.E.

Zhilyaeva Tatyana Vladimirovna ${ }^{1,2 *}$

Chekanina Oksana Mikhailovna ${ }^{1}$, Rukavishnikov Grigory Victorovich ${ }^{2}$, Blagonravova Anna Sergeevna ${ }^{1}$, Mazo Galina Elevna ${ }^{2}$

1 - Federal State Budgetary Educational Institution of Higher Education "Privolzhsky Research Medical University", Ministry of Health of the Russian Federation, 10/1 Minin and Pozharsky sq., Nizhny Novgorod, 603005 Russia;

2 - Federal State Budgetary Institution "V.M. Bekhterev National Medical Research Center for Psychiatry and Neurology", Ministry of Health of the Russian Federation, 3 Bekhtereva st., St. Petersburg, 192019 Russia

*- Corresponding author.

\section{Abstract}

Despite a large amount of data on the association of folate metabolism disturbances with different aspects of schizophrenia, the role of the MTHFD1 1958 G>A polymorphism in this disorder is barely studied. The aim of this study was to 
assess the distribution of alleles and genotypes frequencies of MTHFD1 $1958 \mathrm{G}>\mathrm{A}$ in patients with schizophrenia and healthy controls and to study the association of allele/genotype carriage of this SNP with biochemical markers of one-carbon metabolism and with the severity of schizophrenia symptoms. Methods: In 57 patients with schizophrenia and 37 healthy volunteers the carriage of alleles/genotypes of the MTHFD1 $1958 \mathrm{G}>\mathrm{A}$ and biochemical markers of folate metabolism disturbances were evaluated. Clinical symptoms of schizophrenia and the severity of extrapyramidal side effects of therapy were assessed in patients. Results: an association of the wild GG genotype with schizophrenia was shown (GG versus AG / AA: $\chi 2=7.31 ; \mathrm{p}=0.007$ ). The serum folate level in carriers of the wild genotype GG is lower (in all participants $\mathrm{p}=0.024$, in patients $\mathrm{p}=0.10$ ), and the level of cobalamin in this subgroup is higher (in all participants $\mathrm{p}=0.047$, in patients $\mathrm{p}=0.091$ ) than in carriers of other genotypes. Patients carrying the $G$ allele had less severe negative symptoms $(p=0.0041)$ and extrapyramidal side effects of antipsychotics $(\mathrm{p}=0.054)$, than patients with AA genotype. The age of psychosis manifestation is the later, the more wild alleles $\mathrm{G}$ are present in the genotype $(\mathrm{p}=0.00195)$.

\section{Keywords:}

schizophrenia; MTHFD1 1958 G> A; folate metabolism; methylation; tetrahydrobiopterin.

\section{Introduction}

The hypothesis of one-carbon metabolism (hereinafter OCM) disturbances has become one of the most discussed biochemical theories in the etiopathogenesis of schizophrenia. This hypothesis also reflects the multifactorial nature and complexity of the disease. D. Wang et al. meta-analysis (2015) of 26 independent studies, showed a significant association of plasma folate deficiency with schizophrenia (Wang, D. et al., 2016). Earlier studies have shown that homocysteine levels are higher in patients with schizophrenia compared to healthy controls (Ayesa-Arriola, R. et al., 2012). Moreover, meta-analyzes have confirmed the causal link between hyperhomocysteinemia and schizophrenia (Numata, S. et al., 2015; Nishi, A. et al., 2014). For today, a large amount 
of data has been accumulated that made it possible to conduct large-scale meta-analyzes and prove more frequent carriage of the minor $\mathrm{T}$ allele of the single nucleotide polymorphism (SNP) of the folate cycle key enzyme methylenetetrahydrofolate reductase (hereinafter MTHFR) $677 \mathrm{C}>\mathrm{T}$ in schizophrenic patients compared with healthy subjects (10,069 patients with schizophrenia and 13,372 controls) (Yadav, U. et al., 2016).

Furthermore, from the areas of medicine (eg. obstetrics, cardiology), where disorders of folate metabolism have long been studied as an etiopathogenetic factor of various conditions, it is known that in addition to one single SNP, a number of other genetic factors contribute to the disturbances of folate metabolism, providing no less powerful impact on biochemical parameters than SNP MTHFR $677 \mathrm{C}>\mathrm{T}$. And among such factors SNP of methylenetetrahydrofolate dehydrogenase-1 (MTHFD1) enzyme $1958 \mathrm{G}>\mathrm{A}$ (rs2236225) can be named.

The MTHFD1 gene is located on chromosome 14 (14q24). A common MTHFD1 $1958 \mathrm{G}>$ A polymorphism (Arg653Gln) reduces the enzyme's activity and stability and has been associated with an increased risk of several human diseases, including neural tube defects (Brody, L.C. et al., 2002), congenital heart defects (Christensen, K.E. et al., 2009), unexplained second semester pregnancy loss (ParleMcDermott, A. et al., 2005). The role of this genetic factor in the oncological diseases is also shown in the studies (Moruzzi, S. et al., 2017). The research of this locus have been conducted in relation to cardiovascular diseases, but the results are ambiguous (Ding, Y.P. et al., 2016; García-González, I. et al., 2015). In an experiment it was shown that MTHFD1 is an essential gene in mice and alters biomarkers of impaired one-carbon metabolism (MacFarlane, A.J. et al., 2009).

Methylenetetrahydrofolate dehydrogenase, MTHFD1 is a tri-functional cytoplasmic folate metabolizing enzyme that catalyzes the interconversions of tetrahydrofolate (THF), 10-formyl-THF, 5,10-methenyl-THF, and 5-10 methyleneTHF (Ivanov, A. et al., 2009). The last two are the donor cofactors for de novo purine and pyrimidine and, thus, for the DNA biosynthesis (Hol, F.A. et al., 1998). The MTHFD1 $1958 \mathrm{G}>\mathrm{A}$ polymorphism resides in the 10-formylTHF synthetase domain 
and results in the substitution of an arginine for a glutamate at residue 653 in the protein product.

In schizophrenia, the role of this locus is not entirely clear. Kempisty B. et al. (2007) found that MTHFD1 1958AA or 1958AG genotypes constitute risk factors for schizophrenia with odds ratio $(\mathrm{OR})=2.667$ (95\% $\mathrm{CI}=1.845-3.854 ; \mathrm{P}=0.0001)$. However, this study did not assess the association of SNP with clinical and biochemical parameters, and therefore the biochemical mechanisms of this genetic locus impact on the risks of schizophrenia remain unknown. The authors concluded that it is necessary to reproduce the results in other populations and that further study of this genetic factor role in the pathogenesis of mental disorders is needed. Unfortunately, there were no publications on similar further studies in other populations, as well as on the studies of this locus association with biochemical and clinical parameters in schizophrenia. In studies of the effect of SNPs allelic variants of folate cycle enzymes on schizophrenia negative symptoms, this allele was also not presented, despite the fact that the role of other genetic loci affecting folate metabolism in the negative cluster of schizophrenia symptoms was shown (Roffman, J.L. et al., 2011). Also their influence on the results of folate augmentation of antipsychotic therapy of schizophrenia has been shown (Roffman, J.L. et al., 2013). Considering data from related medical areas, it is necessary to assess the influence of this factor not only on the risk of schizophrenia, but also on the risks of folate deficiency in this disease, as well as on the development of different clusters of symptoms.

The aim of this work was to assess the distribution of alleles and genotypes frequencies of MTHFD1 $1958 \mathrm{G}>\mathrm{A}$ in patients with schizophrenia and healthy controls. The second goal was to study the association of allele / genotype carriage of this SNP with biochemical markers of one-carbon metabolism, as well as the severity of schizophrenia symptoms and treatment side-effects.

\section{Methods}

The study was conducted in compliance with the principles of the Helsinki Declaration of the World Medical Association. The protocol and informed consent for the study participants were approved by Local Ethical Committee No. 1 of Privolzhsky 
Research Medical University (03/13/2019). All the study participants gave a written informed consent for participation and data processing.

The criteria for inclusion of patients in the study were: confirmed schizophrenia diagnosis using the Mini International Neuropsychiatric Interview for Diagnostic and Statistical Manual, Version 5 (M.I.N.I. for DSM-5); patient's ability to give informed consent to participate in the study; no history of any vitamins and antioxidants intake for a month before inclusion in the study. The demographic characteristics of the studied groups are presented in Table 1.

Table 1. Studied groups characteristics.

\begin{tabular}{|l|l|l|l|}
\hline & Patients $(\mathrm{n}=57)$ & Healthy control $(\mathrm{n}=37)$ & $\mathrm{p}-$ level \\
\hline $\begin{array}{l}\text { Male / female, } \\
\text { abs. }\end{array}$ & $34 / 23$ & $16 / 21$ & $\begin{array}{l}\chi 2=1,81 ; \\
\mathrm{p}=0,18\end{array}$ \\
\hline $\begin{array}{l}\text { Age, years } \\
\text { Me [Q1; Q4] }\end{array}$ & $29[22 ; 34]$ & $25[22 ; 32]$ & $\begin{array}{l}\mathrm{Z}=0,92 ; \\
\mathrm{p}=0,35\end{array}$ \\
\hline
\end{tabular}

Footnote: Me [Q1; Q4] - median and interquartile range.

All patients received antipsychotic treatment (doses in chlorpromazine equivalent - $170[100 ; 300] \mathrm{mg}$, median and interquartile range) and were under the supervision of psychiatrists in day-time care $(n=13)$ or inpatient $(n=41)$ departments, as well as on an outpatient basis $(n=3)$. The average duration of the disease since the time of onset was $21.5 \pm 2.8$ months $(\mathrm{M} \pm \mathrm{SE})$.

All patients were examined using PANSS (Positive and Negative Syndrome Scale, Kay, S.R. et al., 1987). Evaluation of extrapyramidal side effects (EPS) was performed with the section for antipsychotic extrapyramidal side effects of the UKU side effect rating scale (version “The UKUSERS-Clin”, Lingjaerde, O. et al., 1987).

Blood samples were collected in the morning after night fasting (at least 6 hours) from the cubital vein. 
Molecular diagnostics of MTHFD1 1958G>A (rs2236225) SNP were performed in all participants by the method of polymerase chain reaction (hereinafter PCR) with allele-specific primers and subsequent detection in real-time. Test systems for the isolation of DNA from white blood cells "DNA-Express blood" (made by Scientific and Production Company "Liteh", Russia) and the set for allele definition of the single nucleotide polymorphism rs2236225 (made by LLC “TestGene”, Russia) were used.

BH4, B12, folates and Hcy were evaluated in all participants. Quantitative determination of BH4 serum level ( $\mathrm{pg} / \mathrm{ml}$ ) was performed by competitive enzymelinked immunosorbent assay (ELISA) using a CEG421Ge (Cloud-Clone Corp) kit. The results were assessed on a Sunrise spectrophotometer, Tecan (Austria). The determination of folate $(\mathrm{ng} / \mathrm{ml})$ and cobalamin $(\mathrm{B} 12, \mathrm{pg} / \mathrm{ml})$ serum levels was performed by the method of chemiluminescent microparticle immunoassay (CMIA, Architect, Abbott lab.S.A.). Homocysteine (Hcy) serum concentration $(\mu \mathrm{mol} / \mathrm{L})$ was measured with a Cobas analyzer (Roche Diagnostics) using an enzymatic assay. The blood samples were blinded with regard to the study group (patients or healthy).

Statistical analysis of the data was performed $t$ on the basis of MS Excel and Statistica 10.0. The normal distribution of data in the samples was assessed using the Shapiro-Wilk test (Shapiro-Wilk W-test). Since the distributions of the obtained data in the studied cohorts differed from the normal one, nonparametric criteria were used: Mann-Whitney U-test for comparing 2 groups (hereinafter MWU-test), Kruskal-Wallis test for comparing 3 groups (KW-test); $\chi 2$ test (with Yates' correction when comparing frequencies in two groups); Spearman's rank test ( $\rho$ ) was used to assess the correlations. The data are presented using the median and interquartile range (Me [Q1; Q3]). Differences were considered statistically significant at $\mathrm{p}<0.05$.

\section{Results}

As can be seen from Table 2, the distribution of genotypes significantly differed in patients and healthy controls, mainly due to the prevalence of wild allele $G$ in homozygous form in patients (this allele doesn't reduce the function of the MTHFD1 
enzyme). Therefore, genotype GG could be considered as a risk genotype for schizophrenia.

Table 2. Distribution of different alleles and genotypes of

MTHFD1 $1958 \mathrm{G}>\mathrm{A}$ in patients with schizophrenia and healthy controls.

\begin{tabular}{|c|c|c|c|c|c|}
\hline \multirow[t]{2}{*}{$\begin{array}{l}\text { Alleles and genotypes of } \\
\text { MTHFD1 1958G>A }\end{array}$} & \multicolumn{2}{|c|}{ Patients } & \multicolumn{2}{|c|}{$\begin{array}{l}\text { Healthy } \\
\text { controls }\end{array}$} & \multirow[t]{2}{*}{ p-level } \\
\hline & Abs. & $\%$ & Abs. & $\%$ & \\
\hline AA & 15 & 26 & 17 & 46 & \multirow{3}{*}{$\begin{array}{l}\chi 2=8,99 \\
p=0.012\end{array}$} \\
\hline GA & 18 & 32 & 15 & 41 & \\
\hline GG & 24 & 42 & 5 & 13 & \\
\hline $\begin{array}{l}\text { A in homo- and heterozygous } \\
\text { form }\end{array}$ & 33 & 58 & 32 & 87 & \multirow{2}{*}{$\begin{array}{l}\chi 2=7,31 ; \\
p=0.007 \\
\text { OR=4,66; } \\
95 \% \text { CI }[1,58 ; \\
13,70]\end{array}$} \\
\hline G homozygous form & 24 & 42 & 5 & 13 & \\
\hline Total number & 57 & 100 & 37 & 100 & - \\
\hline
\end{tabular}

For further statistical analysis of associations with biochemical and clinical parameters alleles and genotypes of MTHFD1 1958 G>A were coded as follows: wild allele (G) - 1, polymorphic allele (A) - 0; genotypes: GG - 2, GA - 1, AA - 0.

In all study participants (entire sample) the more wild alleles were in the MTHFD1 1958G>A locus and therefore the better the function of the MTHFD1 enzyme, the lower were the plasma folate levels and the higher the B12 levels $(\mathrm{p}<0.05)$ (Table 3). In patients the results were similar, but statistically insignificant, probably due to the smaller number of observations in the sample. Furthermore, the levels of 
BH4 in patients increased with an increase in the number of wild G alleles in the genotype (approaching the level of statistical significance).

Table 3. Correlation analysis of the MTHFD1 1958G>A genotypes carriage with biochemical parameters.

\begin{tabular}{|l|r|r|r|r|l|l|l|l|l|}
\hline & \multicolumn{4}{|l|}{ All participants } & \multicolumn{3}{l|}{ Patients } & \multicolumn{2}{l|}{ Healthy controls } \\
\cline { 2 - 11 } & \multicolumn{1}{l|}{$\mathrm{n}$} & $\rho$ & $\mathrm{p}$ & $\mathrm{n}$ & $\rho$ & $\mathrm{p}$ & $\mathrm{n}$ & $\rho$ & $\mathrm{p}$ \\
\hline BH4 & 94 & 0,013 & 0,898 & 57 & 0,20 & 0,14 & 37 & $-0,18$ & 0,29 \\
\hline folates & 94 & $-0,23$ & $0,024 *$ & 57 & $-0,22$ & 0,10 & 37 & 0,008 & 0,96 \\
\hline Hcy & 94 & 0,10 & 0,319 & 57 & 0,02 & 0,91 & 37 & $-0,007$ & 0,97 \\
\hline B12 & 94 & 0,21 & $0,047 *$ & 57 & 0,23 & 0,09 & 37 & 0,22 & 0,19 \\
\hline
\end{tabular}

Footnote: MTHFD1 1958 G>A genotypes codes: GG - 2, GA - 1, AA -0; * $\mathrm{p}<0,05$.

The values of the studied biochemical parameters in the examined participants with different genotypes of MTHFD1 $1958 \mathrm{G}>\mathrm{A}$ are presented in Figure 1 (all participants) and Figure 2 (patients). 
Figure 1. The values of the studied biochemical parameters in the examined participants with different genotypes of MTHFD1 1958 G> A (0 - AA; 1 - GA; 2 - GG), the entire sample.
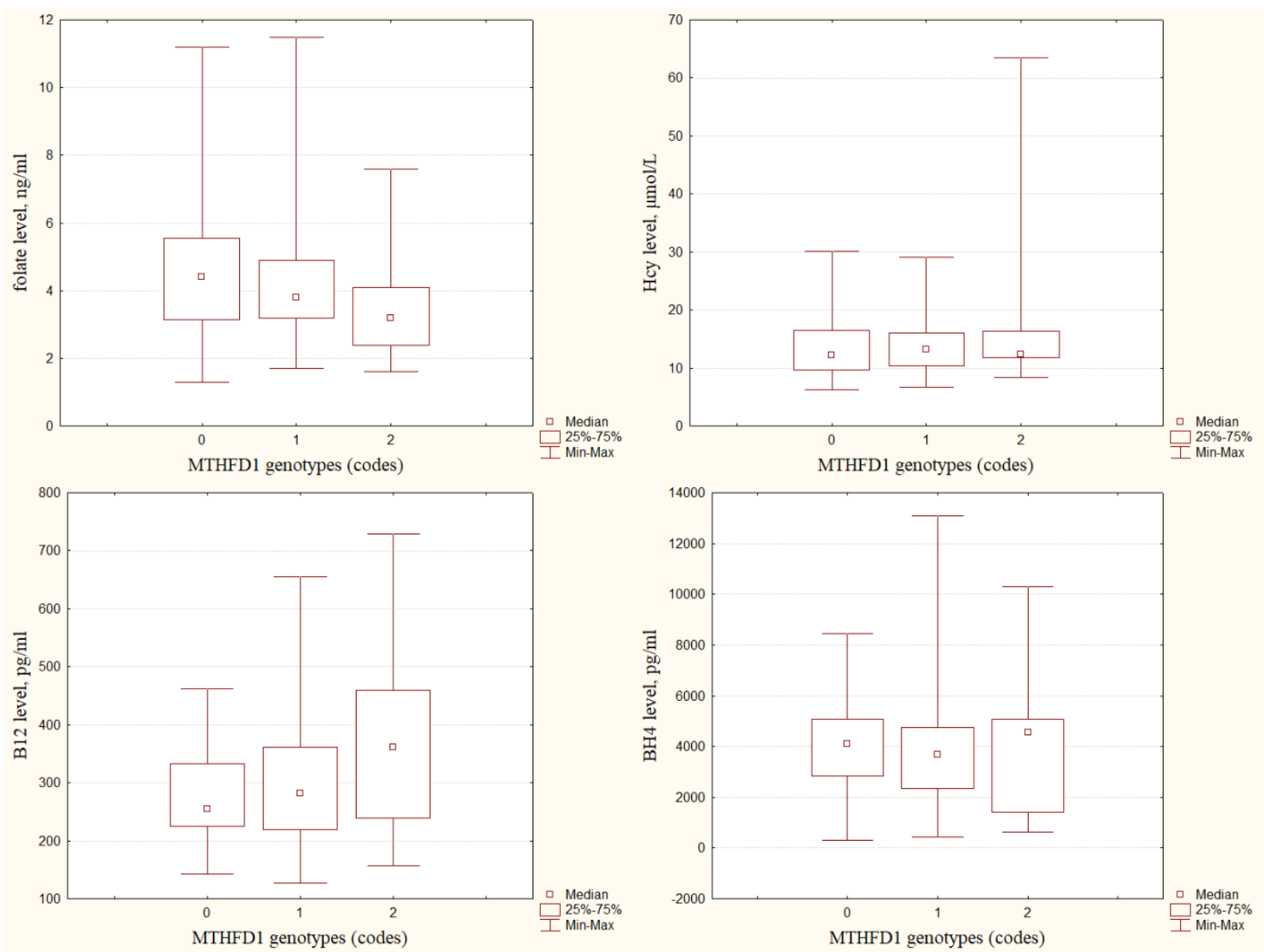
Figure 2. The values of the studied biochemical and clinical parameters in patients with different genotypes of MTHFD1 $1958 \mathrm{G}>\mathrm{A}$ (0 - AA; 1- GA; 2 - GG).
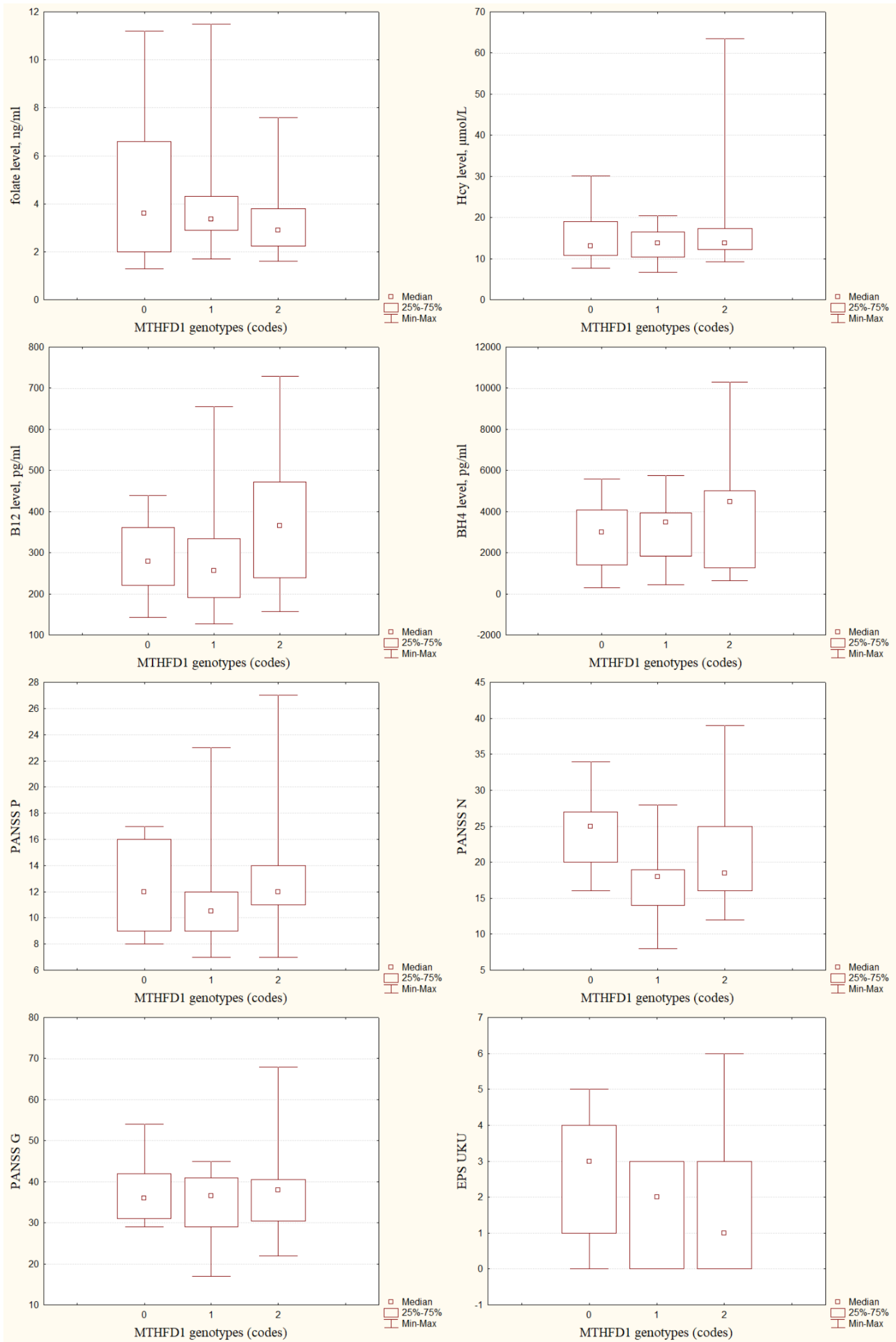
The association of the genotypes carriage with clusters of clinical symptoms (according to the subscales of PANSS) and the severity of side effects of antipsychotics was also analyzed in patients (Table 4).

Table 4. Analysis of the carriage of MTHFD1 1958G> A genotypes and alleles with clinical parameters in patients.

\begin{tabular}{|l|l|l|l|l|}
\hline \multirow{2}{*}{} & \multicolumn{2}{|l|}{$\begin{array}{l}\text { Genotypes (AA/ AG/GG, } \\
\mathrm{n}=57), \mathrm{KW} \text {-test }\end{array}$} & \multicolumn{2}{|c|}{$\begin{array}{r}\text { Allele G present (GG+AG, n=42) } \\
\text { vs. allele G absent (AA, n=15), } \\
\text { MWU-test }\end{array}$} \\
\cline { 2 - 5 } & $\mathrm{H}$ & $\mathrm{p}$ & $\mathrm{Z}$ & $\mathrm{p}$ \\
\hline PANSS P & 2,53 & 0,28 & 0,0091 & 0,99 \\
\hline PANSS N & 9,74 & $0,0077^{* *}$ & $-2,87$ & $0,0041^{* *}$ \\
\hline PANSS G & 1,05 & 0,59 & $-0,47$ & 0,64 \\
\hline EPS UKU & 3,71 & 0,15 & $-1,93$ & 0,054 \\
\hline
\end{tabular}

Footnote: $* *-p<0,01$.

As it can be seen from Figure 3, the lower was the enzyme function (with the higher serum folate levels, the lower serum $\mathrm{BH} 4$ levels and the higher negative symptoms scores according to Figure 2), the earlier was the onset of the disease.

Figure 3. The age of schizophrenia manifestation in patients with different genotypes of MTHFD1 $1958 \mathrm{G}>\mathrm{A}(0$ - AA; $1-\mathrm{GA} ; 2$ - GG). 


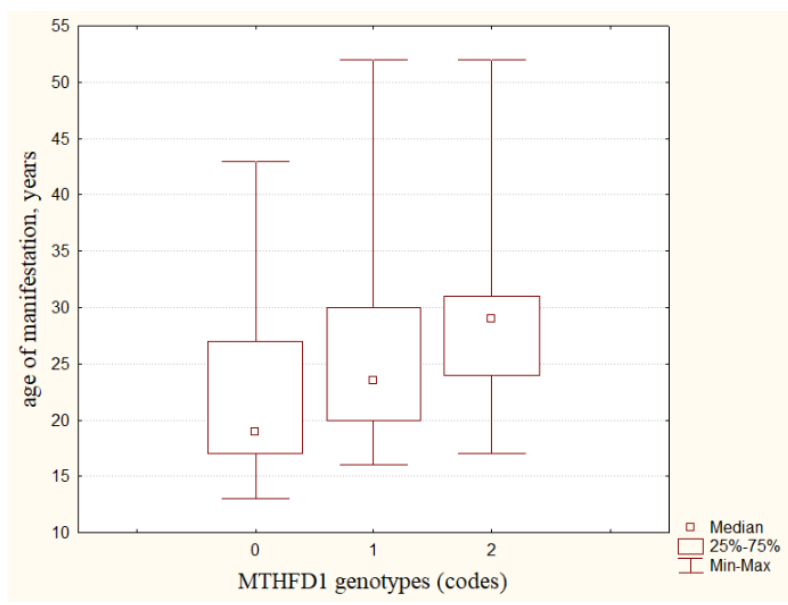

Footnote: $\mathrm{H}=7,88, \mathrm{p}=0,0195,(\mathrm{KW}$-test; 0 - $\mathrm{AA} ; 1$ - $\mathrm{GA} ; 2$ - GG).

\section{Discussion}

As first appears the obtained results are paradoxical. The carriage of a polymorphic allele A (genotype AA), which reduces the enzyme function, is associated with the lower risks of schizophrenia, the higher levels of plasma folate both in patients and in the whole sample, but at the same time with more severe negative symptoms of schizophrenia and antipsychotic extrapyramidal side effects in patients. However, a more thorough analysis of the literature data on the studied genetic locus, as well as on the association of its genotypes with biochemical parameters, allows to give a rational explanation of the results.

According to the literature data, the main function of the MTHFD1 enzyme is participation in the synthesis of purine bases (Hol, F.A. et al., 1998), as well as in the thymidylate cycle of the pyrimidine bases synthesis (Field, M.S. et al., 2016). The blockade of nucleic acid synthesis is considered as the main reason for the increased risk of congenital malformations in the case of impaired MTHFD1 enzyme function.

However, it can be assumed that in individuals with the carriage of the MTHFD1 polymorphic allele A when folate consumption for the synthesis of nucleic acids decreases (turnover in the cycle of purine synthesis decreases), this increases their level in plasma / serum (which is consistent with our data).

Our data that the serum folate levels in carriers of the polymorphic allele A of MTHFD1 $1958 \mathrm{G}>\mathrm{A}$ are higher than in carriers of the wild genotype GG at this locus are consistent with the data of other authors. Thus, according to Cabo R. et al. (2015,

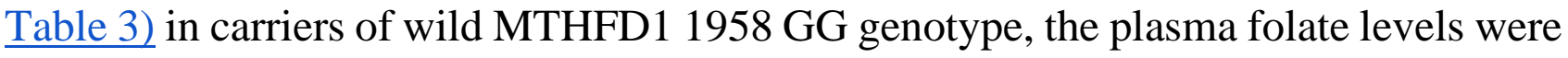


lower than in carriers of polymorphic allele A in both homo- and heterozygous forms, initially and after folate intake. There are no other publications showing the association of the MTHFD1 genotypes with the level of plasma/serum folate in the available literature. At the same time, data have been published that the enzymes thymidylate synthase and MTHFD1 compete with the MTHFR enzyme for the methyl groups of plasma methylfolate (Blom, H.J. et al., 2006, Figure 3).

Thus, it can be assumed that the synthesis of nucleic acids is a competitive cellular process for homocysteine methylation to methionine (Figure 4). This explains why decreased MTHFD1 function in carriers of the polymorphic allele A is a protective factor with regard to the risk of schizophrenia. For schizophrenia the impaired MTHFR function, decreased plasma folate levels and hyperhomocysteinemia are considered proven risk factors (Wang, D. et al., 2016; Ayesa-Arriola, R. et al., 2012; Numata, S. et al., 2015; Nishi, A. et al., 2014; Yadav, U. et al., 2016). The normal MTHFD1 function contributes to the decrease in the functional activity of MTHFR due to competition for the shared substrate, as it can be seen in Figure 4 and is consistent with data of Blom H.J. et al. (2006). 
thymidylate

synthesis

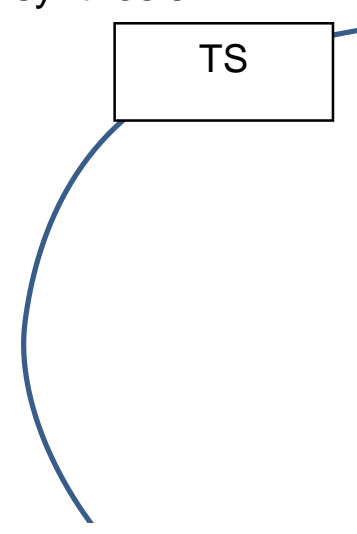

DHF

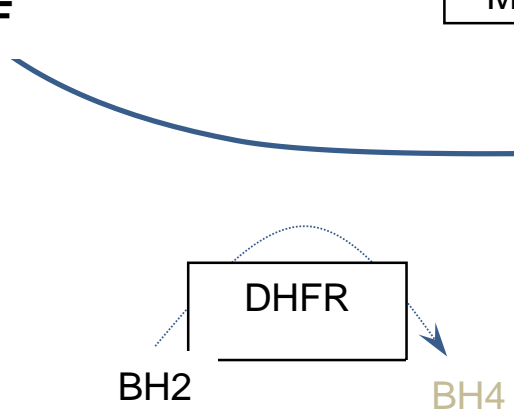

Figure 4. Exchange of pterins, competing cycles in the case of MTHFD loss of function. Methylenetetrahydrofolate reductase (MTHFR) competes with thymidylate synthase (TS) and methylenetetrahydrofolate dehydrogenase (MTHFD) for the onecarbon units of 5-Methylenetetrahydrofolate (5-MethyleneTHF). SAM - Sadenosylmethionine; MTR - methionine synthase; 5-MeTHF - 5Methyltetrahydrofolate; Hcy - homocysteine; DHF - dihydrofolate; DHFR - DHF reductase; SHMT - serine hydroxymethyltransferase; THF - tetrahydrofolate; $\mathrm{BH} 2$ dihydrobiopterin; BH4 - tetrahydrobiopterin.

It can also be seen in Figure 4 that the cycle of purine synthesis with the participation of MTHFD1 is competitive (also for a shared substrate) for the thymidylate cycle, another biochemical process for the synthesis of nucleic bases. In this cycle another enzyme of the folate cycle, dihydrofolate reductase (DHFR), is involved. In the context of this work, it is important that DHFR is involved in BH4 
resynthesis, the level of which, according to our data, is reduced in carriers of polymorphic MTHFD1 allele A. Dihydrofolate and dihydrobiopterin (BH2) are substrates for the common DHFR enzyme. A decrease in the turnover in the purine synthesis cycle with a decrease in MTHFD1 function promotes an increase in turnover in the thymidylate cycle, which is competitive for the substrate. This may contribute to an increase in the turnover of dihydrofolate (DHF). Since DHF competes with BH2 for a common enzyme, it reduces BH4 resynthesis. This hypothesis was confirmed experimentally in a study by Whitsett J. et al. (2013), in which it was shown that folic acid, which is converted to dihydrofolate, reduces BH4 resynthesis in human endothelial cells. This may explain the decrease in plasma BH4 levels among carriers of the polymorphic allele A of MTHFD1 1958G>A in patients, which increases dihydrofolate turnover.

Moreover, there are data that oxidized forms of folates (folic acid and dihydrofolate) can inhibit the activity of dihydropteridine reductase, another enzyme that resynthesizes BH4 (Sánchez-del-Campo, L. et al., 2010). Thus, at least two mechanisms are known (competitive inhibition of DHFR and noncompetitive inhibition of DHPR) by which dihydrofolate is able to block BH4 resynthesis. Since BH4 is a key cofactor in the synthesis of a number of neurotransmitters, in particular monoamines, and its level is reduced in patients carrying the polymorphic allele A of MTHFD1 $1958 \mathrm{G}>\mathrm{A}$, this may be a link explaining the increase in severity of negative symptoms and antipsychotic extrapyramidal side effects in carriers of this allele. The association of the BH4 level with the severity of negative symptoms in our study does not reach statistical significance. An increase in the sample size is required to confirm this hypothesis. The ability of folic acid to block BH4 resynthesis has important consequences for the practical use of this form of folate in schizophrenia. The blockade of $\mathrm{BH} 4$ resynthesis by excess dihydrofolate can lead to an increase in the severity of the negative symptoms, which requires special evaluation.

The ability of folic acid to block BH4 resynthesis has important consequences for the practical use of this folate form in schizophrenia: the blockade of BH4 resynthesis by excess dihydrofolate can lead to an increase in the severity of the negative symptoms, which requires special study. 
In carriers of the wild MTHFD1 1958G>A genotype the contradiction between decreased folate levels, increased risk of schizophrenia, but decreased severity of negative symptoms may indicate that the mechanisms involved in the manifestation of schizophrenia psychotic and positive symptoms may be different from the mechanisms involved in the development of negative and cognitive symptoms of schizophrenia. Hypothetically violation of methionine synthesis and methylation processes for the positive symptoms and BH4 deficiency / impaired synthesis of neurotransmitters (including dopamine) for the negative symptoms. This is consistent with the T. Crow hypothesis of negative / positive subtypes of schizophrenia (Crow, T., 1981). In support of this hypothesis, the age of schizophrenia onset in carriers of the low-functional MTHFD1 1958A allele with more severe negative symptoms in our sample is statistically significantly lower than in carriers of the wild MTHFD1 $1958 \mathrm{G}$ allele. This data is consistent with T. Crow's data on the negative subtype of schizophrenia.

Earlier in one of the studies, results were obtained that are consistent with our data. Polymorphism of the MTHFR 677C $>$ T gene (allele T) (functionally corresponds to the carriage of the wild allele MTHFD1 1958G) is involved in the pathogenesis of schizophrenia subtype with rapid and stable therapeutic response to typical antipsychotics with a good long-term prognosis and a favourable therapeutic result (Joober, R. et al., 2000).

The risk of schizophrenia in disturbances of folate metabolism may be associated with impaired methionine synthesis and utilization of homocysteine, hyperhomocysteinemia, impaired methylation processes, which is associated with a direct deficiency of folates (donor of methyl groups). This was repeatedly mentioned in a number of publications (Wang, D. et al., 2016; Ayesa-Arriola, R. et al., 2012; Numata, S. et al., 2015; Nishi, A. et al., 2014).

In different patients with a combination of certain genetic factors both $\mathrm{BH} 4$ and methylation deficiency can occur, or only one of them. The MTHFD1 1958G>A polymorphism is unique because it affects in an alternative way two groups of mechanisms, and therefore, at first glance, contradictory results were obtained in our study. However, these results make it possible to understand that the risk of manifestation of psychosis and the risk of a poor prognosis (severity of negative 
symptoms) in schizophrenia may be due to different mechanisms. Disturbances in the function of different enzymes of the folate cycle can affect these mechanisms dichotomously as in the case of the MTHFD1 enzyme and synergistically, as in the case of the MTHFR enzyme. It is possible that the synergistic effect of the MTHFR deficiency on negative symptoms and schizophrenia manifestation at the same time was the reason why the data on the association of MTHFR 677C $>$ T SNP with schizophrenia were obtained earlier than others.

If the data from this study are confirmed in other populations and larger-scale studies, it will be possible to conclude that violations of DNA synthesis in the pathogenesis of schizophrenia do not play such an important role as disorders of methylation.

\section{Funding sources and conflict of interest}

The study was supported by Russian Foundation for Basic Research (grant numbers: 19-015-00420 A - biochemical laboratory and clinical investigations; 20015-00301 A - molecular diagnostics of MTHFD1 1958G>A (rs2236225) SNP) and by Privolzhsky Research Medical University (assessment of extrapyramidal side effects). The funding source had no role in study design, analysis, interpretation, and any other processes in the paper.

All the authors declare no conflicts of interests. 


\section{References}

Wang, D., Zhai, J.-X., \& Liu, D.-W. (2016). Serum folate levels in schizophrenia: A $\begin{array}{llll}\text { meta-analysis. } & \text { Psychiatry } & \text { Research, } & \text { 235, }\end{array}$ doi:10.1016/j.psychres.2015.11.045

Ayesa-Arriola, R., Pérez-Iglesias, R., Rodríguez-Sánchez, J. M., Mata, I., Gómez-Ruiz, E., García-Unzueta, M., ... Crespo-Facorro, B. (2012). Homocysteine and cognition in first-episode psychosis patients. European Archives of Psychiatry and Clinical Neuroscience, 262(7), 557-564. doi:10.1007/s00406-012-0302-2

Numata, S., Kinoshita, M., Tajima, A., Nishi, A., Imoto, I., \& Ohmori, T. (2015). Evaluation of an association between plasma total homocysteine and schizophrenia by a Mendelian randomization analysis. BMC Medical Genetics, 16(1). doi:10.1186/s12881-015-0197-7

Nishi, A., Numata, S., Tajima, A., Kinoshita, M., Kikuchi, K., Shimodera, S., ... Ohmori, T. (2014). Meta-analyses of Blood Homocysteine Levels for Gender and Genetic Association Studies of the MTHFR C677T Polymorphism in Schizophrenia. Schizophrenia Bulletin, 40(5), 1154-1163. doi:10.1093/schbul/sbt154

Yadav, U., Kumar, P., Gupta, S., \& Rai, V. (2016). Role of MTHFR C677T gene polymorphism in the susceptibility of schizophrenia: An updated meta-analysis. Asian Journal of Psychiatry, 20, 41-51. doi:10.1016/j.ajp.2016.02.002

Brody, L. C., Conley, M., Cox, C., Kirke, P. N., McKeever, M. P., Mills, J. L., ... Swanson, D. A. (2002). A Polymorphism, R653Q, in the Trifunctional Enzyme Methylenetetrahydrofolate Dehydrogenase/Methenyltetrahydrofolate Cyclohydrolase/Formyltetrahydrofolate Synthetase Is a Maternal Genetic Risk Factor for Neural Tube Defects: Report of the Birth Defects Research Group. The American Journal of Human Genetics, 71(5), 1207-1215. doi:10.1086/344213

Christensen, K. E., Rohlicek, C. V., Andelfinger, G. U., Michaud, J., Bigras, J.-L., Richter, A., ... Rozen, R. (2009). The MTHFD1 p.Arg653Gln variant alters enzyme function and increases risk for congenital heart defects. Human Mutation, 30(2), 212-220. doi:10.1002/humu.20830 
Parle-McDermott, A., Pangilinan, F., Mills, J. L., Signore, C. C., Molloy, A. M., Cotter, A., ... Brody, L. C. (2005). A polymorphism in the MTHFD1 gene increases a mother's risk of having an unexplained second trimester pregnancy loss. MHR: Basic Science of Reproductive Medicine, 11(7), 477-480. doi:10.1093/molehr/gah204

Moruzzi, S., Guarini, P., Udali, S., Ruzzenente, A., Guglielmi, A., Conci, S., ... Friso, S. (2017). One-carbon genetic variants and the role of MTHFD1 1958G>A in liver and colon cancer risk according to global DNA methylation. PLOS ONE, 12(10), e0185792. doi:10.1371/journal.pone.0185792

Ding, Y. P., Pedersen, E. K. R., Johansson, S., Gregory, J. F., Ueland, P. M., Svingen, G. F. T., ... Nygård, O. K. (2016). B vitamin treatments modify the risk of myocardial infarction associated with a MTHFD1 polymorphism in patients with stable angina pectoris. Nutrition, Metabolism and Cardiovascular Diseases, 26(6), 495-501. doi:10.1016/j.numecd.2015.12.009

García-González, I., Solís-Cárdenas, A. de J., Flores-Ocampo, J. A., Alejos-Mex, R., Herrera-Sánchez, L. F., \& González-Herrera, L. J. (2015). Polimorfismos G894T del gen NOS3 y G1958A del gen MTHFD1 y riesgo de cardiopatía isquémica en Yucatán, México. Clínica e Investigación En Arteriosclerosis, 27(2), 64-73. doi:10.1016/j.arteri.2014.07.002

MacFarlane, A. J., Perry, C. A., Girnary, H. H., Gao, D., Allen, R. H., Stabler, S. P., ... Stover, P. J. (2009). Mthfd1 Is an Essential Gene in Mice and Alters Biomarkers of Impaired One-carbon Metabolism. Journal of Biological Chemistry, 284(3), 1533-1539. doi:10.1074/jbc.m808281200

Ivanov, A., Nash-Barboza, S., Hinkis, S., \& Caudill, M. A. (2009). Genetic Variants in Phosphatidylethanolamine N-methyltransferase and Methylenetetrahydrofolate Dehydrogenase Influence Biomarkers of Choline Metabolism When Folate Intake Is Restricted. Journal of the American Dietetic Association, 109(2), 313-318. doi:10.1016/j.jada.2008.10.046 
Hol, F. A., Put, N. M., Geurds, M. P., Heil, S. G., Trijbels, F. J., Hamel, B. C., ... Blom, H. J. (2008). Molecular genetic analysis of the gene encoding the trifunctional enzyme MTHFD (methylenetetrahydrofolate-dehydrogenase, methenyltetrahydrofolate-cyclohydrolase, formyltetrahydrofolate synthetase) in patients with neural tube defects. Clinical Genetics, 53(2), 119-125. doi:10.1111/j.1399-0004.1998.tb02658.x

Kempisty, B., Sikora, J., Lianeri, M., Szczepankiewicz, A., Czerski, P., Hauser, J., \& Jagodzinski, P. P. (2007). MTHFD 1958G>A and MTR 2756A >G polymorphisms are associated with bipolar disorder and schizophrenia. Psychiatric Genetics, 17(3), 177-181. doi:10.1097/ypg.0b013e328029826f

Roffman, J. L., Brohawn, D. G., Nitenson, A. Z., Macklin, E. A., Smoller, J. W., \& Goff, D. C. (2011). Genetic Variation Throughout the Folate Metabolic Pathway Influences Negative Symptom Severity in Schizophrenia. Schizophrenia Bulletin, 39(2), 330-338. doi:10.1093/schbul/sbr150

Roffman, J. L., Lamberti, J. S., Achtyes, E., Macklin, E. A., Galendez, G. C., Raeke, L. H., ... Goff, D. C. (2013). Randomized Multicenter Investigation of Folate Plus Vitamin B12Supplementation in Schizophrenia. JAMA Psychiatry, 70(5), 481. doi:10.1001/jamapsychiatry.2013.900

Kay, S. R., Fiszbein, A., \& Opler, L. A. (1987). The Positive and Negative Syndrome Scale (PANSS) for Schizophrenia. Schizophrenia Bulletin, 13(2), 261-276. doi:10.1093/schbul/13.2.261

Lingjærde, O., Ahlfors, U. G., Bech, P., Dencker, S. J., \& Elgen, K. (1987). The UKU side effect rating scale: A new comprehensive rating scale for psychotropic drugs and a cross-sectional study of side effects in neuroleptic-treated patients. Acta Psychiatrica Scandinavica, 76(s334), 1-100. doi:10.1111/j.16000447.1987.tb10566.x

Field, M. S., Kamynina, E., \& Stover, P. J. (2016). MTHFD1 regulates nuclear de novo thymidylate biosynthesis and genome stability. Biochimie, 126, 27-30. doi:10.1016/j.biochi.2016.02.001 
Chittiboyina, S., Chen, Z., Chiorean, E. G., Kamendulis, L. M., \& Hocevar, B. A. (2018). The role of the folate pathway in pancreatic cancer risk. PLOS ONE, 13(2), e0193298. doi:10.1371/journal.pone.0193298

Cabo, R., Hernes, S., Slettan, A., Haugen, M., Ye, S., Blomhoff, R., \& Mansoor, M. A. (2015). Effect of genetic polymorphisms involved in folate metabolism on the concentration of serum folate and plasma total homocysteine (p-tHcy) in healthy subjects after short-term folic acid supplementation: a randomized, double blind, crossover study. Genes \& Nutrition, 10(3). doi:10.1007/s12263-015-0456-4

Blom, H. J., Shaw, G. M., den Heijer, M., \& Finnell, R. H. (2006). Neural tube defects and folate: case far from closed. Nature Reviews Neuroscience, 7(9), 724-731. doi:10.1038/nrn1986

Whitsett, J., Rangel Filho, A., Sethumadhavan, S., Celinska, J., Widlansky, M., \& Vasquez-Vivar, J. (2013). Human endothelial dihydrofolate reductase low activity limits vascular tetrahydrobiopterin recycling. Free Radical Biology and Medicine, 63, 143-150. doi:10.1016/j.freeradbiomed.2013.04.035

Sánchez-del-Campo, L., Chazarra, S., Montenegro, M. F., Cabezas-Herrera, J., \& Rodríguez-López, J. N. (2010). Mechanism of dihydrofolate reductase downregulation in melanoma by 3-O-(3,4,5-trimethoxybenzoyl)-(-)-epicatechin. Journal of Cellular Biochemistry, 110(6), 1399-1409. doi:10.1002/jcb.22656

Crow, T. (1981). Positive and Negative Schizophrenia Symptoms and the Role of Dopamine. British Journal of Psychiatry, 139(3), 251-254. doi:10.1192/bjp.139.3.251

Joober, R., Benkelfat, C., Lal, S., Bloom, D., Labelle, A., Lalonde, P., ... Rouleau, G. A. (2000). Association between the methylenetetrahydrofolate reductase $677 \mathrm{C} \rightarrow \mathrm{T}$ missense mutation and schizophrenia. Molecular Psychiatry, 5(3), 323-326. doi:10.1038/sj.mp.4000724 